\title{
Evaluation of Primary Health Care in Brazil: A Literature Review
}

\section{Tatiane Baratieri*}

Professor of Universidade Estadual do Centro-Oeste-Unicentro, Brazil

\begin{abstract}
This study aimed to analyze the scientific literature on the aspects and/or positive and negative impacts of Primary Health Care $(\mathrm{PHC})$ in Brazil. There was conducted a literature search on the Lilacs database, finding nine articles analyzed by categorical content. The data revealed positive impacts of PHC in Brazil: best performance of the FHS than to Traditional Basic Units, users recognize the $\mathrm{PHC}$ as the gateway of the health system, increasing the professional/user relationship and municipalities those invest in innovative strategies attention. The challenges for the consolidation of PHC excel in studies: classified in most cities studied as regular/partially effective, incipient/unsatisfactory, little impact on improving the health conditions of the population, incipient social participation, difficulties in organizing the PHC, professional devaluation. It is concluded that there are incipient positive impacts of PHC, requiring changes, such as increased planning, organization and qualification, to the consolidation of PHC in Brazil. Studies which evaluate the $\mathrm{PHC}$ are extremely important for the reorganization of the health system and overcoming the challenges that still exist in the primary level of attention.
\end{abstract}

Keywords: Primary health care; Health evaluation; Brazil

\section{Introduction}

In 1978, occurred the Alma-Ata Conference, which proposed the Primary Health Care (PHC) as a strategy to expand the service to all segments of society, making use of techniques that were not only clinics, but with an epidemiological and social standpoint $[1,2]$.

At the end of the 1980s the health system in Brazil begins a process of reform of the health sector, and in 1986 in the Brazilian political democratization process, was held the VIII National Health Conference, which proposed an administrative reform and unification of institutions and healthcare service in a unique Ministry of Health, which would be responsible for the conduct and management of the entire health policy [1].

However, the landmark was the Brazilian Federal Constitution of 1988, which made real the ideal to build the Unified Health System (SUS - in Portuguese) from the moment when was proposed to guarantee universal access to health care, exceeding the proposed hitherto existing and introducing a new definition of health, conceptualized as a right. Moreover, contemplated levels of health care, allowing new structuring of health services to prioritize collective and preventive actions, without prejudice to the individual and curative actions, even to the predominant moment [3].

It is noted that Primary Care (PC) and PHC are synonymous, but in this paper we report on the PHC terminology to be broadcast internationally. In Brazil, in the context of the NHS, the PHC is defined as the set of actions of health, individual and collective, which includes the promotion and protection of health, disease prevention, diagnosis, treatment, rehabilitation and health maintenance. It is developed through the exercise of management and sanitary democratic and participatory practices, under the form of teamwork, driven to populations of welldefined territories, for which it is bared the responsibility for health, considering the dynamics existing in the territory in which these people live; the uses of high complexity and low density technologies, which should solve the health problems of greater frequency and relevance in their territory. It is the preferred contact users with health systems. It is guided by the principles of universality, accessibility and coordination of care, bond and continuity, comprehensiveness, accountability, humanization, equity and social participation. The PHC considers the subject in its uniqueness, complexity, and completeness in the sociocultural integration and seeks to promote their health, prevention and treatment of diseases and harm reduction or suffering that may compromise their ability to live healthily [4].
The PHC can be evaluated taking into account their four attributes: attention to the first contact, longitudinality, comprehensiveness, and coordination. Attention to first contact means accessibility and use of health services for each new problem for which people seek health care, and the service is accessible only if the user realizing it as such, reflecting on their use. The longitudinality refers to the existence of a regular source of care and its use over time, with key professional / user link through interpersonal ties and mutual cooperation. The full impact on the provision of all health care services that users need, including referral to more complex levels, while the health care team to identify the health needs of the population in order to direct the actions. The attribute of coordination of care needs ways to continuity of care provided, as well as recognition of the problems [5].

It is of utmost importance to evaluate the PHC, for the purpose of identifying the successes and limitations under the first level of care, in order to formulate policies and direct the actions of health professionals, for that primary care is increasingly determined. It is emphasized that the evaluation only adds value when information is produced on behalf of institutional improvement and professional [6].

\section{Objective}

Reviewing the scientific literature on the aspects and/or positive and negative impacts of PHC in Brazil.

\section{Method}

This work is a bibliographic study, which seeks to explain a problem through theoretical literature references in documents. The literature survey was conducted on the Virtual Health Library (BIREME), in the Lilacs (Latin American Literature in Health Sciences) database considered the main area of the Brazilian health, and the data collection took place in the month of January 2013.

*Corresponding author: Tatiane Baratieri, Professor of Universidade Estadual do Centro-Oeste -Unicentro, Brazil, Tel: (42) 98105804; E-mail: baratieri.tatiane@gmail.com

Received October 24, 2013; Accepted December 18, 2013; Published December 20, 2013

Citation: Baratieri T (2013) Evaluation of Primary Health Care in Brazil: A Literature Review. Primary Health Care 3: 144. doi:10.4172/2167-1079.1000144

Copyright: ( 2013 Baratieri T. This is an open-access article distributed under the terms of the Creative Commons Attribution License, which permits unrestricted use, distribution, and reproduction in any medium, provided the original author and source are credited. 
For the removal of articles, the keywords "primary care to health", "basic health care", "health assessment" by the group of descriptors was performed as follows were used: primary care to health and health evaluation, and basic health care and health evaluation.

As a criteria for the selection of the sample, there were selected articles published in national and international journals, since the survey has been conducted in Brazil and articles that address the issue of evaluation of Primary Health Care/Basic Health Care services and pointing positive and negative aspects of $\mathrm{PHC}$ in Brazil; articles published in the last ten years (2003-2012).

The present study has the following exclusion criteria: no publications that are configured in scientific articles, ie, dissertations, theses, abstract, etc.; articles that do not address the positive and negative aspects of the evaluation of PHC services; articles that are outside the period established for the study (last 10 years).

Thus, it was performed the intersection of the descriptors "primary health care" and "health assessment", and found 537, and after reading the titles and abstracts of articles found, considering the inclusion and exclusion criteria, 22 articles remained. When crossing the descriptors "primary health care" and "health assessment", 650 articles were found, and who met the criteria of the study were the same as those found with the previous intersection.

Subsequently, the 22 selected articles were read in their entirety to fulfill faithfully the protocol elaborated analysis, and remained for analysis only nine articles, which indeed showed positive and negative aspects of PHC in Brazil.

To guide the analysis of the data there was created a spreadsheet containing: author, title, year, and journal published; objective of the study, type of study, and main results.

After the found results there was analyzed using categorical content analysis, which has conducted the pre-analysis by fluctuating readings of all data collected that were configured in the corpus analyzed, enabling the formulation of interpretations and initial questions. Then, there was performed the exhaustive reading of the material, its coding, enumeration, sorting and aggregation. Finally the interpretation and categorization of the results was performed before the identification of units of interest, the commonalities between them, and inferences, allowing discuss the interrelations between the results of the articles found [7].

The discussion happened through the comparisons between data from different articles found in the literature review, and to discuss with studies conducted in other countries in South America, in order to better understand the situation in Brazil.

\section{Results and Discussion}

The material found showed that from the nine articles found, five were held in municipalities with more than 100,000 inhabitants [8-12], and are bound to the Baseline Studies Project Expansion and Consolidation of the FHS. In this context, it is noteworthy that the literature that addresses the organizational assessment and performance of primary care services in Brazil is scarce.

\section{Positive Impacts of PHC in Brazil}

The articles in the study highlighted the importance of the FHS for consolidation of PHC [8-14] reality that strongly evidenced in countries of the region, such as Chile [2].
The FHS is considered responsible for priority attention to users resident in critical areas of socio-epidemiological risk [11]. Thus, the literature shows that the performance of the FHS is better than the traditional services $[8,12,15]$.

It is important to emphasize the study [15] of the State of São Paulo, it was evidenced that the health professionals who act in the context of family health assesses best issues involving bond, and cast of services, family and community oriented focus, than those who work at BHUs. Another study shows that the programmatic actions for comprehensive care and group activities occur most frequently in the FHS than in the traditional model [8], Furthermore, the FHS has better assessment regarding the definition of the geographical area and territory map $[8,11]$ and staff participation in activities in the catchment area.

The studies of Facchini et al. [8] and Camargo et al. [10] addressed issues involving organizational dimension of health services, investigated the PHC in FHS and BHU in the South and Northeast of Brazil, showing that these management practices over the South are: coordination meetings with district or BHU and the Municipal Health Council, the production of management reports and the Primary Care Information System (SIAB - in Portuguese) and monitoring of resources programmed by group of procedures. In the Northeast "workshops, training courses, continuing education activities, meetings with the Municipal Health Council, the coordination of other municipalities and district were referred" [8].

A research carried out in 31 municipalities of Minas Gerais and Espírito Santo, found that these governance is favorable in the majority of municipal managers ordered the expenditure of municipalities. Furthermore, the study showed favorable direction for implementing innovative programs on its own initiative the municipality and intersectoral initiatives [10].

A study conducted in 15 municipalities of Santa Catarina characterized these as appropriate (nine) and non-appropriate (six) due to the FHS coverage and quality of the teams, showing the following positive results for PHC, regarding the reduction in hospitalizations between years 1999 and 2004 for ambulatory care sensitive conditions: downward trend in the number of hospitalizations for diabetes mellitus and pneumonia in older than 60 , in municipalities classified as appropriate; reduction in hospitalizations for pneumonia in children under five in both suitable and non-suitable [13].

The study showed that there were positive changes in reducing some rates of hospitalization for ambulatory care sensitive conditions, which may be associated with the provision of PHC by FHS of quality, and that in municipalities with better care of their family reflected in changes significantly more positive. In this sense, "suggests that the expansion of coverage FHS facilitated access of the population to the services of this level of attention, and that while the classification of attention received contributed to the reduction in hospitalizations for selected causes" [13].

Based on the presented data, the studies confirm the purpose of the FHS is the main way to improve the PHC in Brazil, which leads to increasing discussions about the growing need to expand their coverage in order to enhance positive impacts on health the population.

A study conducted in Bolivia shows that the provision of integrated services within the APS, can significantly improve the health of children [16], which strengthens the claim that quality services in primary care have a positive impact on population health.

Another task that is directly related to positive evaluation of PHC 
is the qualification and offer continuing education and/or continuing healthcare to the professionals. This can be proved by the study of Castro et al. [12], which showed that having expertise in the area of $\mathrm{PHC}$ and offer continuing education for the service are associated with better performance of PHC [12].

The consolidation of the FHS depends heavily on the skills of both managers (advanced training in public health and previous management experience) as professionals (updated on life cycles and programmatic areas of PHC; introductory course to the FHS, specializations and residences in health family) [11] in addition, the study showed that there is investment in the training area (regional, municipal schools for training in public/family health, partnerships with the organs and teaching-service integration).

Regarding the articles about the user, it was identified that there is an increasing recognition of the role of $\mathrm{PHC}$ as the gateway, users perceive and evaluate positively the growth of the link between professional / user $[11,15]$, satisfaction with the work of household and Community Health Agent $[11,17]$, location of family health unit next to the home visit and solving [11].

It is noteworthy that Ibañez et al. [15] pointed out in his study that user's satisfaction is better when it comes to the direct care of the issues surrounding the most profound changes in culture and behavior of services.

Accordingly, there were identified articles that approach the user satisfaction is still incipient in the evaluation studies of PHC, in which Szwarcwarld et al. [9] recognize that the studies that consider user opinion are extremely important to set goals and set priorities in health care.

It should be noted that the evaluative studies of PHS analyzed present positive impacts still timid, and, therefore, it is important to highlight positive experiences presented by some authors.

In a research conducted in Campinas / Sao Paulo, where innovative strategies (teams organized by PSF model, regular weekly meetings for the preparation of therapeutic projects and discussion of clinical cases, the presence of mental health support at least fortnightly and developing joint consultations and discussions were evaluated cases) in the organization of PHC with respect to mental health, identified that a health team of primary care achieved positive results with the implementation of these strategies, in which there was involvement of the whole team, proposals for joint assistance between Professional Center Psychosocial Care (CAPS) and BHUs, which impacted on greater responsibility in cases treated [17].

Another prominent study conducted by Rocha et al. (2008) in 21 municipalities in the Brazilian Northeast, identified in three cities as innovative experiences living pharmacy (herbal medicine), caravan Smile (educational activity for prevention of dental caries), gym city (area of bodily practices in public squares), therapy Community (meetings involving promotion of mental health professionals and users), enlarged FHS (integrated team of specialists), among others, demonstrating greater range of actions from the perspective of care [11].

Based on the above, it is important to pay attention to the experiences of success within the PHC, in addition to the positive impacts it has achieved in Brazil in order to devise strategies / policies more efficient to meet the population, given that PHC is recognized as a strategy to reorganize the health system of many countries in South America $[16,18,19]$.

\section{Challenges to the consolidation of PHC in Brazil}

It can be seen in the subject area that impacts previously discussed and related to evaluation of PHC positives are still incipient. Upon follow-up discussions, this category presents the aspects that still need to change in order to achieve consolidation of PHC. Initially an overview is presented with the clearest findings of articles in order to reflect on the conditions of supply of primary care services in Brazil.

A study conducted in 21 municipalities in Northeast Brazil with the aim of evaluating the experiences of implementation of the Family Health Strategy with a focus on inductions of PROESF, highlights that as the political-institutional dimension most were classified as regular (12) and nephropathy (5), regarding the organization of care was also prevalence of regular (9) and incipient classification (8), on the full care most municipalities was considered with incipient (15) [11]

In this direction, a survey conducted in 15 municipalities of Paraíba showed that with regard to the Organization of the APS the municipalities were mostly considered unsatisfactory (5) and intermediate (8), so that only two municipalities obtained a satisfactory rating.

In Sao Paulo a study was conducted to evaluate the performance of care in PHC services, which present satisfactory results for: gateway, link (or longitudinality) and professionals. However, the dimensions for access, coordination, community-oriented, family focused services and cast the results indicated poor attention [15].

A study in Porto Alegre / Rio Grande do Sul, the PHC services in the municipality: Basic Health Unit (BHU), the Family Health Strategy (FHS); Health Center-School (CSEM - linked to the State Department of Health) and Health Service Community (HSC - linked to the federal public institution) showed that the HSC was considered the best service in the General PHC score, and BHUs had the lowest score. It should be emphasized that $65 \%$ of the municipality receives the services of $\mathrm{PHC}$ through BHUs [12]. It is also important to note that the study revealed that "Access to First Contact" was the attribute of PHC who got lower scores in such services [12].

Another highlight of the study of Szwarcwarld et al. [9]. conducted in four municipalities of Rio de Janeiro, which found that the worse socioeconomic conditions, the worse the coverage and quality of care [9], a result which appears contradictory on the purposes of APS, in particular the ESF in relation to compliance priority.

Corroborating the data presented so far, the study of Ibanez et al. [15] showed that user satisfaction with PHC services is lower than in municipalities with less qualified, i.e they are less satisfied with the location of the health care access and health professionals [15]. Thus, that study found that user satisfaction is classified as intermediate in relation to the basic care of the municipalities surveyed.

Other studies investigating the users' satisfaction were identified difficulties of scheduling with specialists / referrals, lack of disclosure of groups, long waits for care, lengthy queues results of examinations, difficult access, poor listening [11,17].

A study conducted in Peru, as well as Brazilian literature points to the need to improve the PHC in order to increase user satisfaction. This study showed that users are satisfied with geographical accessibility, infrastructure, service hours, and ancillary tests; data are contrary to those found in studies of Brazil. You referred to the attention received, waiting time in emergency and planned actions, the study showed low satisfaction. Thus, about the attributes of the PHC, are apparent needs 
for improvement in continuity of care, attention to first contact and completeness [18].

In a study of 15 municipalities of Santa Catarina characterized these as appropriate (nine) and non-appropriate (six) as the FHS coverage and quality of the teams, showing the following negative regarding the PHC results regarding the reduction in hospitalizations between the years 1999 and 2004 for ambulatory care sensitive conditions: there was an increase of hospitalizations for stroke in appropriate counties and increased by the Acute infarction in both classes of municipalities; regarding the hospitalization in children under five by diarrhea no significant reduction in any of the classes of municipalities [13].

Although the study demonstrated some positive aspects presented above, the results prove precarious changes since the beginning of the implementation of the FHS, in particular with respect to the vagueness of policies related to the work process and insufficient qualification of family health teams. Thus, the study evaluates the care provided by teams of FHS as partially effective in controlling the diseases that were selected for the study, and furthermore, "this partial efficacy may be the result of an explicit demand hitherto latent and the FHP undertook to explain and refer to appropriate care" [13].

It is important to emphasize that some studies have brought clear results even in the prevalence of the traditional model / biomedical $[8,10,11,17]$

In the study of Ibañez et al. [15] it was found that some cities studied still have low coverage of FHS and has the care model based on traditional attention. Thus, Rocha et al. highlights that municipalities with more consolidated FHS practices have more comprehensive care, on the other hand municipalities with the health of the fledgling family there is predominant medicines and clinical-care practices [11]. Campbell et al. stresses that the proposal FHS is still far from its consolidation in view of the limited penetration strategies for reorienting the model [10].

The studies also found data considered as hindering of integral care users, such as incipient use of protocols, $[8,11]$ lack of computerization in the workplace, with demand of time for bureaucratic activities [8], difficulty in accommodating [10,17], high demand culture of welfare of the population, professional work overload, lack of intersectionality (use the spaces of school, church by lack of space on the drive) [17], units in precarious situations, excessive prioritization of specific groups, predominantly single-dressing practices, limited practice health promotion [11]

It is for this moment to reflect on the question of completeness, regarded as one of the principles of the NHS (SUS), one of the requirements and evaluation of $\mathrm{PHC}$ and the base for structuring of the FHS, which forward their nonunion goes against the health system currently proposed. Thus, we emphasize the need to overcome the difficulties pointed out by studies, whether through lack of resources, deficiency in healthcare management, deficits in performance / training, among other reasons, so that PHC can fulfill its role.

The studies brought an alarming data, with regard to the principle of social participation data, pointing to the still nascent social control $[10,14]$. Veras and Vianna detached that of the 15 cities surveyed ten mentioned Participation of the Municipal Board of Health, but none mentioned the participation of the directors in the preparation of projects to evaluate actions [14].

A Colombian study indicates the need to improve aspects those involve intersectoral as well as strengthening community participation and improve $\mathrm{PHC}$, and consequently the welfare of the population [20].

Aside from issues involving direct attention to the user in the context of primary care, it is essential to understand aspects of the organizational dimension of health services, given that this dimension is as important as the other dimensions for the proper functioning of PHC.

On this subject the study of Facchini et al. [8] which compared the PHC in the South and Northeast of Brazil, showed difficulties in organizational dimension regarding the decentralization of the management of primary health care especially for the distance of health facilities in relation to the seat of the Municipal Secretariat of Health and the operational difficulties (transport available and trained staff, information technology); Northeast were scored difficulties of communication with the core network, given the complex hierarchy, and precariousness in information technology, a problem also found in the south.

It is noteworthy that few municipalities incorporate the use of health information management, and there is poor quality of this information particularly the SIAB, and in addition, most municipalities do not return the data to the healthcare teams [10]. Corroborating this finding, another study showed that the majority of municipalities surveyed do not use the indicators to evaluate the PHC, and the 15 cities studied, only two managers surveyed were able to define the responsibility for examining the indicators and their realization [14].

Another important aspect to enable the PHC system is the reference and counter, however, as some studies there are difficulties in communication between levels of care $[11,14,17]$. Veras and Vianna to point out that the underlying instruments and counter only one of the 15 municipalities surveyed had adequate tool and definition of reference services [14].

In addition to the aspects shown so far, it is important to highlight other difficulties encountered in studies concerning the organizational dimension: inadequacy of dentistry and pharmacy services; $[9,11]$ inadequate physical infrastructure, lack of territorial $[8,11]$ lack of Municipal Plan [14].

The literature also points out the precariousness of employment contracts of health workers, especially in the FHS, which disqualifies an adequate attention $[8,10,11]$. In this sense, studies also indicate to staff turnover within the PHC $[10,13,17]$, and more than $25 \%$ of the teams are changed in less time than 12 months and less than half (45\%) of doctors FHS teams meet the workload of 40 hours per week [13].

It is worth noting that as important as the work contracts of satisfactory health professionals is the professional qualification, so that these can enhance your work and improve care to the population. However, this is an aspect that needs to be developed within the PHC $[10,13,14]$, also discussed this aspect in international literature [19].

Chile presents similar data with regard to hiring professionals, revealing that physicians of primary care have fixed-term contracts, unstable careers officers with variable remuneration, without clarity about the possibilities of professional development [2]

Apart from the above, improving the quality of $\mathrm{PHC}$ is also related to monitoring and evaluation by professional health managers. However, studies have pointed out that this practice is still in its infancy in the municipalities surveyed $[8,10,11,14]$, which relates to "the need to incorporate the assessment of the management of the health services system, so that it is used in process of decision making" [14]. 
The literature reveals that supervision of health workers is through informative, through the transfer of rules and bureaucratic procedures (usually associated with the billing of SUS or complaints from users and the media) to health professionals and coordinators units, the use of periodic reports for decision making was rare (due to the heavy workload of the teams in the municipalities and the need for capacity building for monitoring and evaluation activities) [8].

In a study of 21 municipalities in the Northeast, only in three were identified the monitoring and evaluation as consolidated practices [11]. In a study with 15 cities in Paraiba, it was revealed that five of them had no standard or routine control and evaluation and there were only eight routines regarding the evaluation of primary care, this being restricted to the measurement of performance indicators, such as the coefficient of Infant mortality and the occurrence of maternal deaths [14].

A research conducted in Argentina points similar data to those found in Brazil, there are deficiencies in that country on the demographic and epidemiological knowledge of the population, low immunization coverage, low levels of health surveillance of children and women [21], impacts on the level of disability PHC, pointing to the need for improvement of the same [19,21].

The PHC in European countries referred to outpatient services first contact members of a system of universal health coverage, unlike what is observed in Latin America, where PHC has low resolution and quality. Thus, the PHC has narrow objectives and covers only some predefined needs of population groups in extreme poverty, with resource usage low technological density, with little or no guarantee of access to secondary and tertiary levels of health care [22].

Based on the above, it is worth noting that the evaluation of policies and programs is essential for public health, contributing to efforts in search of a healthier society and preventing the waste of resources to the implementation of ineffective programs, 8 but studies have shown this evaluation also shows incipient positive impacts, managers need greater commitment to the consolidation of PHC.

\section{Conclusion}

The study showed that the impacts and positive aspects related to evaluation of the PHC are still incipient.

Moreover, PHC has several challenges to overcome, such as the traditional health care, poor quality and effectiveness, little impact on improving the health conditions of the population, fledgling social participation, difficulties in organizing the $\mathrm{PHC}$, professional devaluation, the need to be overcome especially by improving the planning, organization and qualification of the PHC.

The literature also pointed out a similar reality in various aspects involving the PHC in South America, in countries such as Argentina, Bolivia, Chile, Colombia and Peru, showing that Brazil is inserted into a regional reality that requires larger investments, with respect to the first level of health care.

Evaluative studies in this field are of paramount importance in order to identify the strengths and weaknesses and to direct attention to health in Brazil and the region.

\section{References}

1. Rosa Wde A, Labate RC (2005) [Family Health Program: the construction of a new care model]. Rev Lat Am Enfermagem 13: 1027-1034.

2. Campo B (2012) Family health model in Chile and greater resoluteness of primary health care: contradictory or complementary? Medwave 12: e5571.
3. Nascimento MS, Nascimento MAA (2005) The nurse's role in the Family Health Program: an interface between health surveillance and health program action. Cienc Saude Colet 10: 333-345.

4. Ministry of Health (2006) Department of Health Care Department of Primary Care. National policy for primary care. Brasilia, Brazil: Ministry of Health.

5. Starfield B. Primary Care (2002): balancing health needs, services, and technology. UNESCO, Ministry of Health. 726.

6. Felisberto $E$ (2006) From the theory to the formulation of a National Policy of Evaluation in Health: reopening the debate.11: 553-563.

7. Bardin L (2008) Content analysis. Revised and Updated. Lisbon, Portugal: Edn 70.

8. Facchini LA, Piccini RX, Tomasi E, Thumé E, Silveira DS, et al. (2006) Performance of the PSF in the Brazilian South and Northeast: institutional and epidemiological Assessment of Primaty Health Care. Cienc Saude Colet 11669-11681.

9. Szwarcwald CL, Mendonça MHM, Andrade CLT (2006) Primary health care indicators in four municipalities of the State of Rio de Janeiro, 2005: results of a domestic survey in the population. Cienc Saude Colet 11: 643-655.

10. Camargo KR Jr, Campos EM, Bustamante-Teixeira MT, Mascarenhas MT, Mauad NM, et al. (2008) [Political, institutional, and organizational evaluation of primary health care with an emphasis on comprehensiveness]. Cad Saude Publica 24 Suppl 1: S58-68.

11. Rocha Pde M, Uchoa Ada C, Rocha Nde S, Souza EC, Rocha Mde L, et al. (2008) [An assessment of the Family Health Program in municipalities of Northeast Brazil: old and new challenges]. Cad Saude Publica 24 Suppl 1: S69-78.

12. Castro RC, Knauth DR, Harzheim E, Hauser L, Duncan BB (2012) [Quality assessment of primary care by health professionals: a comparison of different types of services]. Cad Saude Publica 28: 1772-1784.

13. Elias E, Magajewski F (2008) Primary Health Care in the south of Santa Catarina: analysis of admissions for ambulatory care-sensitive conditions, in the 1999-2004 period. Rev Bras Epidemiol 11: 633-647.

14. Veras CLSM, Vianna RPT (2009) Performance Based on the Evaluation of Primary Healh Care Organizational Aspects of Municipalities in the State of Paraíba, Brazil, Epidemiol. Serv Saúde 18: 133-140.

15. Ibañez N, Rocha JSY, Castro PC, Ribeiro MCSA, Forster AC, et al. (2006) Care performance assessment of primary health care services in the State of São Paulo. Ciênc Saúde Coletiva 11: 683-703.

16. Perry HB, Shanklin DS, Schroeder DG (2003) Impact of a community-based comprehensive primary healthcare programme on infant and child mortality in Bolivia. J Health Popul Nutr 21: 383-395.

17. Onocko-Campos RT, Campos GW, Ferrer AL, Corrêa CR, Madureira PR, et al (2012) Evaluation of innovative strategies in the organization of Primary Health Care. Rev Saude Publica 46: 43-50.

18. Cuba-Fuentes M, Jurado G, Estrella E (2011) Attributes of Primary Care and patient satisfaction degree on a primary care facility. Rev Med Hered 224-229.

19. Stolkiner A, Comes Y, Garbus P (2011) Scope and potential of Primary Healthcare in Argentina. Cien Saude Colet 16: 2807-2816.

20. Chávez GBM, Sierra ROLM, Lopera MMM, Montoya TPC (2004) The Basic Health Care Plan (PAB) in decentralized municipalities in Colombia. Rev Fac Nac Salud Pública 22: 77-89.

21. Marin GH, Silberman M, Sanguinetti C (2009) The quality of primary care health centres in Buenos Aires, Argentina. Qual Prim Care 17: 283-287.

22. Bursztyn I, Kushnir R, Giovanella L, Stolkiner A, Sterman-Heimann L, et al. (2010) [Notes for studying primary care within the context of segmented health systems]. Rev Salud Publica (Bogota) 12 Suppl 1: 77-88. 